\title{
Impacto positivo del capital social en la gobernanza forestal comunitaria: Un análisis cuantitativo
}

\section{[Positive impact of social capital on community forest governance: A quantitative analysis]}

\author{
Eduardo Carrillo-Rubio 1(D), Stephen J. Morreale 1 (1) y Vanessa Pérez-Cirera 2 (D)
}

${ }^{1}$ Universidad de Cornell, Estados Unidos; ${ }^{2}$ World Wildlife Fund International

\begin{abstract}
Resumen
El capital social es inobservable, pero se infiere por sus efectos en la sociedad. En un contexto de manejo forestal comunitario, evaluamos la influencia del capital social cognitivo y estructural sobre la deforestación en México empleando estudios en terreno y modelación estadística. Examinamos predicciones acerca del efecto de la demografía, educación, etnicidad, género, y geografía sobre el desempeño de la gobernanza comunitaria. Los resultados revelaron que la deforestación disminuyó en bosques aislados, manejados por comunidades mayoritariamente indígenas, con escolaridad superior al promedio, y mayor proporción de hogares con jefatura femenina. Nuestros hallazgos sustentan la hipótesis principal, la cual sostiene que la cohesión y el capital social son determinantes para la gobernanza forestal sustentable. Nuestra metodología aporta un marco analítico transferible, y los resultados fundamentos para diseñar políticas agrarias, de conservación, y desarrollo rural. Recomendamos estudios adicionales para aumentar la certidumbre muestral y el poder explicativo de los modelos.
\end{abstract}

Palabras clave: comunidades rurales, comunidades indígenas, conservación comunitaria, silvicultura, sistemas socioecológicos.

\begin{abstract}
Social capital is unobservable but can be inferred from its effects on society. We evaluated the influence of cognitive and structural social capital on deforestation rates in community forests in Mexico using surveys and statistical modeling. We examined predictions concerning the influence of demography, education, ethnicity, gender, and geography on community-based forest governance outcomes. Results revealed lower deforestation rates in remote forests managed by majority indigenous communities with above-average education levels and a higher proportion of femaleheaded households. Our findings support the main hypothesis, which proposed that sustainable forest governance is determined by social capital and cohesion. Our methodology constitutes a transferrable analytical framework and the results serve as a point of reference for the design of agrarian, conservation, and rural development policies. We recommend additional studies to reduce sampling uncertainty and increase the explanatory power of the models.
\end{abstract}

Keywords: community-based conservation, forestry, rural and indigenous communities, socio-ecological systems.

Contacto: La comunicación sobre este artículo debe ser enviada a Eduardo Carrillo-Rubio, email ec278@cornell.edu

Financiamiento: Esta investigación contó con financiamiento del Programa Premio Chihuahua Vanguardia en Ciencias y Artes 2020, entregado por el gobierno del Estado de Chihuahua, México.

Agradecimientos: Los autores agradecen a José Luis Montes y al equipo de la Consultoría Técnica Comunitaria (CONTEC) por su asistencia durante el período de trabajo en terreno. 


\section{INTRODUCCIÓN}

El capital social es un activo que representa la propensión que tiene una comunidad o grupo de personas de realizar acciones colectivas mutuamente beneficiosas. Las comunidades con altos niveles de capital social tienen más posibilidades de alcanzar mayores niveles de desarrollo social (Dasgupta \& Serageldin, 1999); mientras que aquellas sin éste pueden cultivarlo y mejorar progresivamente sus niveles de bienestar (Krishna, 2002). Al fomentar la capacidad organizativa y la cohesión social, el capital social puede mejorar el desempeño de programas de desarrollo rural (Sorensen, 2000) y de manejo forestal comunitario (Bray, 2013). Como bien intangible, el capital social no se puede observar directamente. Sin embargo, puede manifestarse a través de comportamientos y acciones colectivas como la cohesión social, las normas y valores compartidos entre miembros de una comunidad (i.e., capital social cognitivo), y las instituciones sociales que se derivan de éstas (i.e., capital social estructural) (Krishna \& Uphoff, 1999). Hasta la fecha, el rol directo e indirecto del capital social sobre la gestión comunitaria de recursos naturales no ha sido estudiado cuantitativamente.

México es el lugar ideal para estudiar las relaciones entre el capital social y la tenencia colectiva de la tierra, y evaluar los conceptos teóricos empleados para entender la gobernanza comunitaria de recursos naturales (DiGiano et al., 2013). Durante el siglo $\mathrm{XX}, 60 \%$ del territorio mexicano y $80 \%$ de los bosques fueron entregados a comunidades rurales e indígenas para conformar miles de ejidos (bosques comunales) y comunidades, ambas formas legales de tenencia colectiva de la tierra. Bajo este esquema, grupos de campesinos se convirtieron en propietarios colectivos de un territorio y sus recursos naturales. En el caso de los ejidos, la Ley Agraria establece que la organización interna es responsabilidad de la Asamblea General Ejidal conformada por todos sus miembros. La asamblea es la máxima autoridad democrática del ejido y la encargada de regular el uso de la tierra y los recursos naturales comunales. La asamblea elige a un comisariado ejidal, que incluye la figura de un presidente o presidenta y otros funcionarios electos por votación. El comisariado se encarga de convocar y presidir las reuniones de la asamblea, ejecutar las decisiones tomadas en ella, y gestionar todos los asuntos relacionados con la vida social del ejido. Podría decirse que la asamblea tiene el potencial de ser el exponente máximo de buen gobierno y lograr mayores niveles de equidad, bienestar, y sustentabilidad, pero para que haya una gobernanza democrática efectiva, debe haber cohesión social; y para generar cohesión social debe haber transparencia, rendición de cuentas, inclusión, y participación (Tornos et al., 2012). En este trabajo, analizamos el papel del capital social cognitivo (cohesión social) y estructural (instituciones comunitarias) sobre la gobernanza del bosque dentro de un contexto de manejo forestal comunitario utilizando como área de estudio la Sierra Tarahumara, México. El manejo forestal comunitario se define como un conjunto de acciones relacionadas con el manejo de los recursos forestales que genera oportunidades económicas para los dueños del bosque. La sustentabilidad de estas prácticas está basada en la premisa de que cuando las comunidades adquieren la responsabilidad de manejar el bosque y el derecho 

V. (2021). Impacto positivo del capital social en la gobernanza forestal comunitaria: Un análisis cuantitativo. Revista de Sociología, 36(2), 1-20. https://doi.org/10.5354/0719-529X.2021.65567

a beneficiarse de él, aumenta su interés y los esfuerzos por cuidarlo. Tras varias décadas de ser implementado en México, el modelo de manejo forestal comunitario se ha propuesto como un modelo global de sustentabilidad que promete mejorar la conservación del bosque y reducir la pobreza en zonas rurales (Bray et al., 2005). Pero a pesar de los casos exitosos, también ha habido numerosos fracasos (ver Carrillo-Rubio, 2005; CarrilloRubio et al. 2014; DiGiano et al., 2013; Durán-Medina et al., 2005; Durán et al., 2010). En muchos casos, el manejo forestal comunitario no logra sus objetivos cuando las comunidades carecen del capital social y humano necesario para organizarse en forma efectiva y manejar sustentablemente sus bosques (McShane et al., 2011; Pérez-Cirera \& Lovett, 2006; Westholm et al., 2011).

En estudios del desarrollo, la hipótesis central de la teoría del capital social se basa en la reciprocidad y el mutualismo como el principio fundamental de la vida en sociedad (Portes, 1998). La idea principal es que las relaciones entre la gente son lo más importante en una sociedad, ya que estas conexiones le permiten a la gente construir comunidad, comprometerse con las causas colectivas y el bien común, desarrollar lazos de cooperación y apoyo, y contribuir a hilar el tejido social. Sin las interacciones entre la gente, las conexiones y el tejido social se desvanecen; y con ello, la confianza, la reciprocidad, y la solidaridad que fortalecen los lazos comunitarios. Dentro de este contexto, el capital social puede describirse como un bien acumulado por un individuo o grupo de individuos a partir de la densidad de las conexiones (red social) entre sus miembros. Estas conexiones, a su vez, se rigen por normas de cooperación y valores compartidos (como la solidaridad, la reciprocidad, y la confianza mutua) que brindan numerosos beneficios al grupo; como la capacidad de realizar acciones colectivas mutuamente beneficiosas. La confianza entre los individuos de una comunidad puede extenderse a personas ajenas e instituciones sociales, que eventualmente se pueden transformar en los valores y virtudes de una sociedad (Keeley, 2007). De acuerdo con Burt (2001) y Coleman (1988), el capital social se origina a partir de las redes sociales cerradas, y son aquellas en las que todos los miembros se conocen entre sí y existen lazos de cercanía. A estas redes sociales también se les conoce como redes cohesionadas o densas. Las redes cerradas generan capital social de dos maneras principales. Primero, el acceso y flujo de información. Un miembro de una red cerrada puede adquirir información directamente de cualquier otro miembro. En cambio, en una red menos densa, la calidad de la información se deteriora si se transfiere a través de una cadena de intermediarios. Segundo, en una red cerrada es más fácil vigilar el comportamiento de los demás miembros e implementar sanciones si alguien viola las normas, lo que aumenta la propensión de la gente a confiar en los demás. Es decir, hay mayor confianza en una comunidad con redes cerradas, porque existe asimilación estructural. Esto es, los miembros tienen conocidos en común, por lo que las expectativas y obligaciones que existen entre ellos y ellas son altas. Además, la pérdida de reputación entre los miembros puede tener un costo social alto en una red cohesionada. En cambio, en una red poco densa o abierta, las expectativas y obligaciones son bajas; y al no existir asimilación o forma de acumular reputación, es difícil implementar sanciones efectivas que aumenten y mantengan la confianza entre los miembros. Por otro lado, el capital social puede generar resultados indeseables, como aumentar la inequidad 
en vez de reducirla. Burt (2001) describe como la desigualdad en una comunidad crea subgrupos que desarrollan mayor capital social que otros. Ellos, a su vez, subordinan al resto para su propio beneficio, creando así un círculo vicioso que aumenta las brechas de desigualdad. Cuando esto ocurre, el capital social se convierte en un bien utilitario. Esto es, un recurso que puede ser explotado por los miembros con mayor poder sobre los demás para beneficio personal en desmedro de la comunidad. Esto ocurre cuando existen huecos estructurales, es decir, vacíos en el tejido social debido a la falta de conexiones entre grupos de personas dentro y fuera de la red social de la comunidad. Esta falta de conexión y flujo de información permite que se forme el tipo de capital social utilitario por personas que aprovechan la oportunidad para prestar servicios como intermediarios. La oportunidad de ocupar esos espacios permite que estos individuos se beneficien personalmente de estas conexiones, pasando a ser prestadores de servicios de intermediación. Por ejemplo, un ente capaz de llenar esos vacíos obtendrá conexiones con diferentes segmentos de la red social, lo que le dará acceso a información y a oportunidades exclusivas. Esa persona o grupo de personas podría aprovechar estas conexiones para acumular información y privilegios que después podría utilizar como apalancamiento para su beneficio personal a costa del resto. En el medio rural, estas personas ocuparían posiciones de poder dentro de sus comunidades y lo aprovecharían para enriquecerse a costa de los demás, formando lo que se conoce comúnmente como cacicazgos. Por ejemplo, un líder con mejor educación y dominio del castellano en una comunidad indígena puede negociar con empresas para extraer madera ilegalmente a cambio de compensaciones económicas.

En el contexto de las comunidades rurales e indígenas en México, se ha hipotetizado que la capacidad de organizarse y desarrollar procesos de autogestión exitosos se debe a los altos niveles de capital social emanados de sus estructuras sociales y formas de organización tradicional (Bray et al., 2005; Toledo, 2000). Esta hipótesis, que se ha empleado para tratar de explicar el éxito de numerosos proyectos de desarrollo rural y manejo forestal sustentable en México, aún no ha sido comprobada con estudios empíricos. Sin embargo, existen experiencias en otros países que sustentan estas afirmaciones. Por ejemplo, Krishna y Uphoff (1999) hicieron uno de los primeros análisis cuantitativos del capital social y su relación con el desempeño de programas de desarrollo rural en la India. Desarrollaron un índice de capital social en 64 comunidades rurales para describir sus tejidos sociales, normas, valores, actitudes y creencias compartidas. Estos índices se correlacionaron con el comportamiento colectivo de las comunidades asociados con el capital social (confianza, apoyo mutuo, reciprocidad, y solidaridad), y se encontró que estaban positivamente relacionados con el desempeño de los programas. En Camerún, Brown et al. (2007) encontraron que los patrones de comportamiento asociados con el capital social (confianza, organización, y la aplicación de sanciones) se traducían en mejor manejo forestal. Así como en la sociología de las redes sociales se han identificado la conexión entre las estructuras sociales y la presencia de capital social (Krishna, 2002; Lin, 2002), se ha sugerido que las estructuras sociales (usos y costumbres, organización tradicional) de las comunidades y pueblos mexicanos tienen un papel 
fundamental en el desarrollo de modelos sustentables de desarrollo rural y explotación forestal (Bray et al., 2005; Sarukhárn \& Larson, 2001; Toledo, 2000). También se ha argumentado que la estabilidad de estas estructuras depende de varios factores importantes, incluyendo la tenencia colectiva de la tierra, la etnicidad, y la cultura. La estructura comunitaria y la visión patrimonial del territorio, un rasgo heredado del México prehispánico que se perpetúa hasta la fecha, puede ser explicado por el hecho que muchas de las comunidades rurales mexicanas tienen raíces indígenas. Debido a esto, se ha argumentado que la conexión espiritual y cultural que tienen algunas comunidades con su territorio es lo que les ha permitido desarrollar el capital social y humano necesarios para preservar su capital natural (Anta \& Pérez, 2004; Sarukhárn \& Larson, 2001; Toledo, 2000). En este estudio, evaluamos el papel del capital social en la gestión de recursos forestales colectivos combinando estudios en terreno y modelación estadística. Comenzamos adoptando la definición y clasificación del capital social de Krishna y Uphoff (1999) en dos tipos (estructural y cognitivo). Después, desarrollamos una serie de variables para representar la condición del bosque (variable dependiente), los tipos de capital social, y otras variables independientes. Con esta lista de variables, construimos modelos hipotéticos de deforestación y los evaluamos empleando datos empíricos y modelos estadísticos. Nuestro estudio aporta un análisis comparativo e inferencia estadística acerca de diversas variables y mecanismos causales que explican el impacto del capital social sobre el éxito o el fracaso del manejo forestal comunitario. Este trabajo ofrece la oportunidad de entender mejor el impacto de la heterogeneidad sociocultural y demográfica sobre la gobernanza del bosque, así como de los factores que pueden explicar los cambios en la cubierta forestal dentro y fuera del área de estudio.

\section{MÉTODO}

\section{Caracterización del área del estudio}

La Sierra Tarahumara es parte de la Sierra Madre Occidental que atraviesa el Estado de Chihuahua, perteneciente a la República Mexicana (ver Figura 1). La Sierra Tarahumara abarca 70,000 $\mathrm{km}^{2}$-una extensión comparable a la superficie de Irlanda-y alberga el 15\% de la biomasa forestal de México. La palabra tarahumara se origina en el siglo XVII y es el nombre que los europeos dieron al grupo indígena más numeroso de la zona. Los tarahumaras se llaman a sí mismos rarámuri (Heras, 2007), que es una palabra indígena que significa pies ligeros.
El área de estudio comprende $1,200 \mathrm{~km}^{2}$ de bosques comunales ubicados entre -106.5 y $-107.5^{\circ} \mathrm{E}$ y 27.8 y $28.8^{\circ} \mathrm{N}$. Las altitudes van de los $1,400 \mathrm{~m}$ en los valles intermontanos hasta $2,700 \mathrm{~m}$ en las cumbres más altas. La topografía se conforma por sierras escarpadas y valles disectados por profundos cañones o barrancas. El clima es templado subhúmedo con lluvias en los meses más calurosos del año que coinciden con el verano en el hemisferio norte. La temperatura media oscila entre los $10-14^{\circ} \mathrm{C}$, y la precipitación total anual promedio es de $800 \mathrm{~mm}$. El principal tipo de vegetación lo conforman bosques templados de coníferas y latifoliadas dominados por arboles de pino ponderosa 

gobernanza forestal comunitaria: Un análisis cuantitativo. Revista de Sociología, 36(2), 1-20. https://doi.org/10.5354/0719-529X.2021.65567

(Pinus ponderosa) y roble (Quercus spp). En los parajes de mayor elevación y humedad se desarrollan bosques mesófilos de coníferas con especies de los géneros Abies, Picea, Pinus, y Pseudotsuga (Rzedowski, 2006).

Figura 1. Ubicación de la Sierra Tarahumara, México.

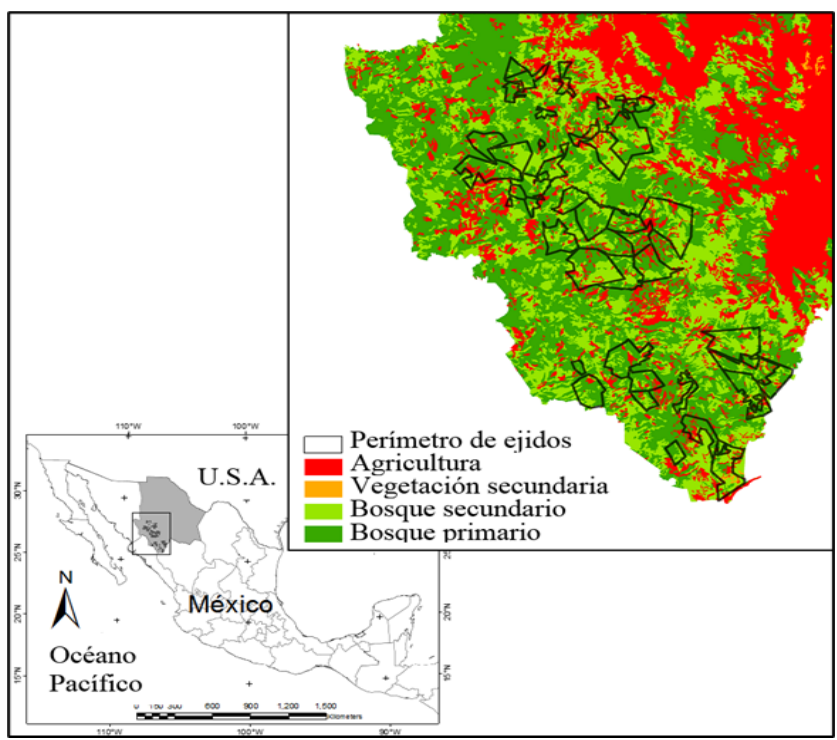

Nota. La parte inferior del mapa indica la ubicación general del área de estudio. El mapa ampliado en la parte superior derecha muestra los tipos de cubierta vegetal y los ejidos seleccionados para este trabajo.

Las principales fuentes de degradación del bosque incluyen el cambio de uso de suelo para agricultura de subsistencia, el sobrepastoreo de ganado doméstico, la deforestación selectiva, y los incendios (Carrillo-Rubio et al., 2014). El modelo de manejo silvícola vigente, basado en el aclareo y regeneración con árboles padre para establecer bosques coetáneos, ha sido fuertemente criticado por reducir la productividad y diversidad de los ecosistemas (Weaver, 2000). La población total de la región se estima en 400,000 habitantes, de los cuales al menos $20 \%$ se identifican como miembros de pueblos originarios. La mayor parte de la población vive en asentamientos rurales pequeños de 1-2,500 personas. En términos de propiedad, el $90 \%$ de la región se encuentra bajo régimen de tenencia colectiva en la forma de ejidos. Menos del 1\% es propiedad pública como parques nacionales y el resto es propiedad privada. Las principales fuentes de ingreso provienen de actividades relacionadas con el aprovechamiento forestal y la ganadería extensiva (Carrillo-Rubio et al., 2014).

\section{Diseño de la muestra}

Para evaluar el efecto del capital social en la gestión de los bosques comunales, desarrollamos un marco analítico y una lista de variables basándonos en la literatura sobre los recursos comunes, la acción colectiva, y la silvicultura comunitaria. Las variables seleccionadas fueron empleadas para diseñar el protocolo de muestreo y construir los modelos estadísticos. Estos modelos representaron nuestras hipótesis relacionadas con el desempeño de la gobernanza comunitaria y los factores multidisciplinarios asociados con la deforestación.

Usamos un muestreo aleatorio estratificado (Zuur et al., 2009) para seleccionar una muestra $(n=44)$ de un universo de ejidos que contaban con permisos de aprovechamiento forestal $(m=$ 128). Este muestreo se utiliza cuando existen estratos o subgrupos en una población y el objetivo del estudio es comparar los estratos entre sí para derivar inferencia estadística acerca de la población total. En este caso, 
estratificamos el total de ejidos en función de tres variables identificadas a priori que influyen sobre el manejo forestal comunitario: (a) el tamaño del grupo; (b) la población indígena, y (c) los ingresos por venta de madera.

Las variables independientes y la dependiente se obtuvieron a partir de fuentes oficiales $\mathrm{y}$ muestreos en terreno. La variable dependiente, definida como la tasa de deforestación (Ha/año), se obtuvo estimando los datos de cobertura vegetal para cada ejido a partir de imágenes satelitales de 1976-2010. Los datos de tenencia de la tierra se obtuvieron de la cartografía elaborada por el Registro Agrario Nacional (RAN) de México. Las bases de datos de la demografía, educación, etnicidad, y nivel socioeconómico a nivel comunidad y hogares provienen de los resultados del censo de población y vivienda del Instituto Nacional de Estadística y Geografía (INEGI) de México.

Para caracterizar el funcionamiento de las asambleas ejidales y describir la importancia económica del bosque, se obtuvieron datos relacionados con la organización institucional de los ejidos y los aprovechamientos forestales a partir de cuestionarios, encuestas, y observaciones estructuradas. Todas las variables independientes, y su relación con el capital social y el estado del bosque, se describen detalladamente en la Tabla 1.

\section{Hipótesis}

La lista de variables nos permitió confrontar varias hipótesis definidas a priori. Por ejemplo, esperábamos que los ejidos con baja tasa de deforestación anual tendrían mayores niveles de capital social cognitivo y, por ende, mayor capacidad de gobernar sus bosques. Consideramos que los ejidos con nula o poca deforestación tendrían mejor desempeño en términos de gestión forestal, rendición de cuentas, y participación democrática en sus asambleas (Pérez-Cirera \& Lovett, 2006; Wexler \& Bray, 1996). Por eso, esperábamos que el efecto de las variables relacionadas con el funcionamiento de la asamblea general y las reglas formales e informales fuera significativo. Con respecto al impacto del capital social cognitivo, nos enfocamos en la cohesión social y la capacidad organizativa de las comunidades representadas por las variables tamaño del grupo y población indígena. Estimamos que los ejidos de mayor población y aquellos sin una mayoría étnica podrían tener dificultades para organizarse en forma efectiva, reflejándose en la condición del bosque (Poteete \& Ostrom, 2004). Esperábamos que una mayor distancia a mercados y centros regionales de población reduciría la probabilidad de deforestación en los ejidos (Nelson \& Hellerstein, 1997). Consideramos que los ejidos con mayor escolaridad promedio tendrían menos deforestación por ser variable indicadora de capacidad técnica (Brown et al., 2007; Wexler \& Bray, 1996). Estimamos que la variable jefatura de hogar femenina estaría negativamente asociada con la conservación del bosque por ser un posible indicador de migración y fragmentación social en los ejidos (Bray et al., 2005). Estimamos que los ingresos por venta de madera incentivarían la conservación del bosque 
en algunos casos (Ostrom, 1990). Esperábamos que la variable modelo comunitario de extracción estaría asociada con menores tasas de deforestación por ser representativa de la cohesión social en el ejido. También que el efecto de las variables inventario de madera en pie y explotación previa del bosque sería nula, pues ambas son indicadoras de gobernanza efectiva y manejo forestal sustentable. Finalmente, predijimos que el precio de la madera en el mercado podría ser un incentivo de doble filo: por un lado, podría motivar a las comunidades a cuidar mejor sus bosques, pero también podría incrementar la tala ilegal.

\section{Análisis estadístico}

Nuestra inferencia se obtuvo empleando índices de información y técnicas multimodelo (Burnham \& Anderson, 2002; Zuur et al., 2009). Este enfoque permite formular y comparar diferentes modelos a partir de las hipótesis del estudio, seleccionar el que mejor se ajusta a los datos, y estimar los parámetros del modelo. El primer paso en este proceso fue determinar si la distribución de la variable dependiente (tasa de deforestación) se distribuía normalmente. Encontramos que la distribución de esta variable se aproximaba a una distribución Poisson con sobredispersión, por lo que adoptamos el modelo lineal generalizado (GLM por sus siglas en inglés) para una distribución binomial negativa.
Después, evaluamos las variables explicativas para verificar que fueran independientes (baja colinealidad) y que tuvieran un sentido social y ecológico contrastado para evitar relaciones espurias.

Después, construimos 14 modelos a partir de nuestras hipótesis para explicar los patrones de deforestación en el área de estudio. Comparamos cada uno de los modelos con los datos, y seleccionamos el que mejor se ajustó a ellos con base en el criterio de información de Akaike (AIC). El índice AIC calcula el nivel de soporte estadístico de un modelo o grupo de modelos según el grado de ajuste a los datos y su complejidad siguiendo el principio de parsimonia. El mejor modelo según el índice AIC es aquel que explica la mayor cantidad de variabilidad con el menor número de variables $K$. Contrastamos los modelos calculando la diferencia entre el mejor modelo y el resto, donde $\triangle \mathrm{AIC}=\mathrm{AICi}-$ mínimo(AIC). Los modelos fueron clasificados en función de su peso relativo de evidencia $w$, el cual indica la probabilidad de que un modelo sea mejor en función de los datos. El valor de $w$ oscila entre $0-1$, donde 1 indica el máximo soporte o poder explicativo. Finalmente, calculamos la importancia relativa para todas las variables predictivas, calculada por la suma de $w$ para todos los modelos que incluían cada variable. Usamos el programa ArcGIS 10.2 para los análisis geoespaciales y la elaboración de mapas, y R para los análisis estadísticos. 
Tabla 1. Descripción de las variables independientes y su impacto esperado sobre el estado del bosque: Aforestación (+), Deforestación (-), y Sin Cambio (o).

Variables relacionadas con el capital social estructural: Instituciones comunitarias que Estado del

regulan los roles y las interacciones sociales con reglamentos y sanciones.

1. Reuniones de Asamblea Anuales (total/año)

Bosque

2. Participación en la Asamblea Asamblea General (porcentaje del total de ejidatarios)

$+$

$+$

3. Decisiones tomadas por mayoría (porcentaje de las decisiones)

Variables relacionadas con el Capital Social Estructural, democracia y rendición de cuentas, y control centralizado sobre el bosque (Pérez-Cirera \& Lovett, 2006; Wexler \& Bray, 1996).

4. Reglas formales y Reglas informales

Las sanciones y otras medidas de control social reducen la tala ilegal (Ostrom, 1990).

Variables relacionadas con el capital social cognitivo: Comportamientos y acciones colectivas como la cohesión social, normas y valores compartidos de una comunidad.

5. Población indígena (tres categorías): 0-25\%, 25-50\%, y >50\% de la población total

La presencia de grupos étnicos puede aumentar la cohesión social y la gobernabilidad; o ser fuente de divisiones intercomunitarias y pérdida de control sobre el manejo del bosque (Pérez-Cirera \& Lovett, 2006).

6. Tamaño del grupo (tres categorías): ejidos con 20-150, 150-280, y >280 miembros

El tamaño del grupo puede tener una relación no-lineal en algunos casos de acción colectiva.

El tamaño efectivo del grupo favorece la organización y acción colectiva eficaz; mientras que $+1-$

los grupos pequeños pueden ser más cohesivos, y por tanto, contar con mayor Capital Social (Poteete \& Ostrom, 2004).

7. Modelo comunitario de extracción (dos categorías): grupos de ejidatarios, o equipos individuales Indicador de fragmentación social. La división de volúmenes de cosecha también reduce el control sobre los recursos y la competitividad económica (DiGiano et al., 2013; Wexler $+1-$ \& Bray, 1996).

Otras variables independientes: Características espaciales, económicas, demográficas, o socioculturales de los bosques comunales.

\section{Distancia a mercados}

La cercanía a centros urbanos acelera la tala legal e ilegal (Nelson \& Hellerstein, 1997).

9. Escolaridad promedio (en años)

Mayor capacidad técnica permite a las comunidades negociar y vigilar como se manejan sus

bosques, dándoles mayor control sobre su uso (Brown et al., 2007; Wexler \& Bray, 1996). 
Tabla 1. Continuación

Otras variables independientes: Características espaciales, económicas, demográficas, o Estado del socioculturales de los bosques comunales.

Bosque

10. Jefatura de hogar femenina (tasa mujeres:hombres)

Indicador de emigración y divisiones comunitarias. La emigración altera las redes sociales, los procesos de toma de decisiones, y reduce el control de la comunidad sobre sus recursos (Bray et al., 2005).

11. Explotación previa del bosque

El tiempo transcurrido en años desde que iniciaron las actividades de extracción en los ejidos. Se espera que el impacto sea nulo si la tasa extracción no ha excedido la tasa de regeneración.

12. Ingresos por venta de madera

Se espera que el interés en la conservación sea proporcional a los ingresos que representa el bosque para los ejidos (Ostrom, 1990).

13. Inventario de madera en pie (estimada en volumen/pies Doyle $\left.{ }^{3}\right)$

El volumen existente no debe variar con el tiempo si la extracción no excede la regeneración.

14. Precio de la madera

El incremento del valor de la madera puede incentivar el manejo sustentable, pero también la tala ilegal. Si los precios son bajos, puede disminuir el interés en cuidar el bosque y la posibilidad de sobreexplotar el bosque para compensar por la pérdida de utilidades.

Nota:Las variables se clasificaron en tres tipos: Variables Relacionadas con el Capital Social Estructural, Variables Relacionadas con el Capital Social Cognitivo, y Otras Variables Independientes.

\section{RESULTADOS}

Los análisis indican que en 2010 más del 15\% del área de estudio se encontraba deforestada, un incremento de casi $100 \%$ con respecto a 1976 . La tasa de deforestación promedio por ejido fue de 42.0 hectáreas anuales. Solo $20 \%$ de los ejidos presentaron ganancias de cobertura arbórea equivalente al $0.14 \%$ de la cubierta forestal en el área de estudio.

El modelo mejor ajustado, con 9 parámetros, tuvo $72 \%$ del peso de evidencia acumulado $w \mathrm{y} \mathrm{AIC}=384.2$. Este modelo fue significativamente mejor que el resto. El segundo mejor modelo, también con 9 parámetros, tuvo $\mathrm{AIC}=388.5$ (4.3 unidades más) y $w=0.08$. De acuerdo con los coeficientes del modelo, los bosques con menor deforestación fueron aquellos ubicados a mayores distancias relativas de los mercados regionales, manejados por comunidades pequeñas (menos de 280 miembros) mayormente indígenas (más del 50\%), con una escolaridad mayor al promedio y la jefatura de los hogares ocupada en su mayoría por mujeres (ver Tabla 2 y Figura 2). 
Tabla 2.

Coeficientes de Regresión Para Todas las Variables Incluidas en el Modelo de Deforestación de 19762010 en la Sierra Tarahumara, México

\begin{tabular}{llll}
\hline Coeficientes & Coef. & Sd & $p^{\text {-valor }}$ \\
\hline Intercepto & 7.7 & 0.870 & $<.001^{* *}$ \\
Población indígena $25-50 \%$ & -0.057 & 0.187 & $<.100$ \\
Población indígena $>50 \%$ & -1.122 & 0.251 & $<.001^{* *}$ \\
Tamaño del grupo $150-280$ & -0.064 & 0.165 & $<.100$ \\
Tamaño del grupo $>280$ & 0.653 & 0.154 & $<.001^{* *}$ \\
Distancia a mercados & -0.101 & 0.024 & $<.001^{* *}$ \\
Escolaridad Promedio & -0.256 & 0.081 & $<.010^{*}$ \\
Jefatura de hogar femenina & -1.490 & 0.540 & $<.010^{*}$ \\
\hline
\end{tabular}

* Significativo al 99\% de confianza. ** Significativo al 99.9\% de confianza

Los coeficientes del modelo para la población indígena 25-50\% y población indígena $>50 \%$ tienen un signo negativo, pero solo ésta última es estadísticamente significativa. Esto indica que la deforestación promedio es menor en ejidos con población mayoritariamente indígena. Los resultados revelan una relación no-lineal entre la tasa de deforestación y tamaño del grupo. En ejidos con 150-280 miembros, la deforestación fue menor comparada con ejidos de menor y mayor tamaño; mientras que ejidos con más de 280 miembros la deforestación fue mucho mayor (coeficiente significativo con signo positivo). Las variables distancia a mercados, escolaridad promedio, y jefatura de hogar femenina fueron estadísticamente significativas con signo negativo. Esto indica que mientras mayor sean estos valores, menor será la deforestación. Por ejemplo, si escolaridad promedio se incrementa en una unidad (año), se esperaría que la tasa de deforestación sea 0.26 veces menor. Comparando ejidos a diferentes distancias del mismo mercado regional, encontramos que por cada unidad (kilómetro) adicional de la variable Distancia a mercados la tasa de deforestación se redujo 0.1 veces. Similarmente, si la jefatura de hogar femenina se incrementara de 1:1 a 2:1 mujeres:hombres, la tasa de deforestación seria 1.5 veces menor. Siete variables tuvieron soporte bajo, y 3 carecieron de soporte en los modelos. El poder explicativo para todas las variables, estimado a partir de la suma del peso relativo de evidencia $W$, aparecen en la Tabla 3.

Figura 2. Coeficientes e Intervalos de Confianza (95\%) Para las Variables Incluidas en el Modelo de Deforestación de 1976-2010

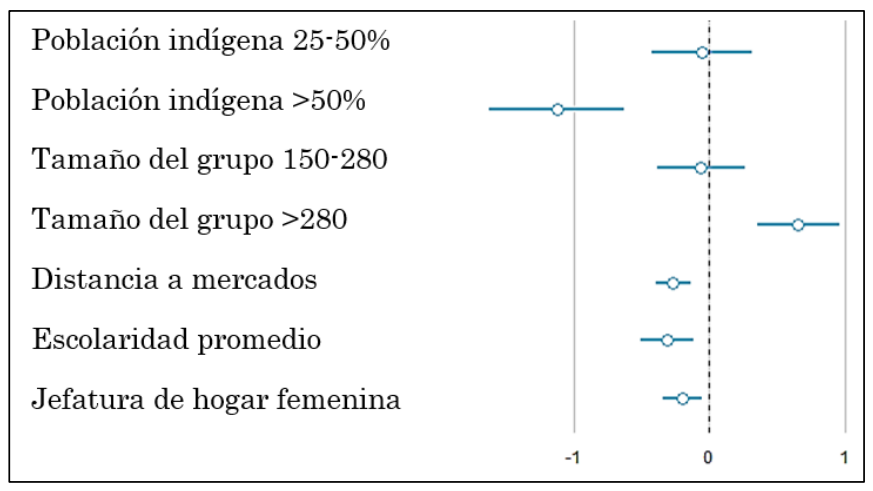

Nota: Las variables fueron estandarizadas para facilitar su interpretación. 
Tabla 3.

Importancia Relativa (Suma $A I C W$ ) y Coeficientes (B) Para Todas las Variables Predictivas Incluidas en los Modelos Evaluados

\begin{tabular}{lcc}
\hline Variable & Suma AIC $w$ & B (Promedio) \\
\hline Distancia a mercados & 0.99 & -0.08 \\
Tamaño del grupo $150-280$ & 0.98 & -0.04 \\
Tamaño del grupo >280 & 0.98 & 0.51 \\
Población indígena 25-50\% & 0.95 & 0.04 \\
Población indígena $>50 \%$ & 0.95 & -0.68 \\
Escolaridad promedio & 0.80 & -0.21 \\
Jefatura de hogar femenina & 0.72 & -1.50 \\
Reglas formales & 0.15 & -0.16 \\
Modelo comunitario de extracción & 0.08 & -0.35 \\
Reuniones de asamblea anuales & 0.08 & -0.27 \\
Explotación previa del bosque & 0.04 & -0.01 \\
Ingresos por venta de madera & 0.03 & 0.14 \\
Inventario de madera en pie & 0.02 & 0.18 \\
Reglas informales & 0.003 & 0.24 \\
\hline
\end{tabular}

\section{DISCUSIÓN Y CONCLUSIONES}

En este artículo, diseñamos un marco analítico interdisciplinario y multiescalar para evaluar el impacto del capital social sobre la gobernanza comunitaria del bosque. Empleamos imágenes de satélite para evaluar los cambios en la cubierta forestal a largo plazo; estudios en terreno para examinar la relación entre las instituciones comunitarias y la condición del bosque; y censos demográficos para explicar las diferencias institucionales intercomunitarias. Aplicamos esta metodología a una muestra de ejidos con estructuras sociales heterogéneas y discutimos los resultados. El modelo mejor sustentado por los datos demostró la significancia estadística de variables comúnmente asociadas con la perdida de bosque como Distancia a mercados (Nelson \& Hellerstein, 1997; Perz \& Skole, 2003; Rodrigues et al., 2009). También pudimos demostrar una relación significativa entre los cambios en la cubierta forestal a largo plazo con las características demográficas, culturales, y educativas de la población.

Una de las variables más importantes fue Tamaño del grupo, pues encontramos que la tasa de deforestación disminuyó en ejidos de 20-280 miembros, pero aumento en ejidos $\geq 280$. Estos hallazgos coinciden con Nagendra (2007), Pérez-Cirera y Lovett (2006), y Poteete y Ostrom (2004), pero contradicen el trabajo de Chhatre y Agrawal (2009), quienes sostienen que la capacidad de gobernanza forestal se incrementa 
conforme aumenta el número de usuarios. Esta última premisa no tiene ningún sustento en la literatura. En antropología, se ha establecido que existe un número óptimo de personas que pueden mantener una unidad social estable y cohesiva a lo largo del tiempo determinada por la capacidad cognitiva (Dunbar, 1992). Hernando et al. (2010) encontraron que la optimización de la unidad social ocurre en grupos que promedian 150 (rango: 100-230) miembros. A medida que aumenta el tamaño del grupo, también la necesidad de establecer más reglas y normas de comportamiento para mantener la estabilidad y la cohesión grupal. Además, Burt (2001) y Coleman (1988) señalan que las redes cohesionadas o densas son las que están asociadas con mayores niveles de capital social, puesto que la cohesión facilita el flujo de información y la verificación del cumplimiento de las normas y valores establecidos. $\mathrm{Al}$ aumentar el tamaño del grupo, estas cualidades se pierden. Estas conclusiones coinciden con nuestros resultados y validan nuestras premisas de que la sustentabilidad del manejo forestal comunitario depende en buena parte de la cohesión y el capital social comunal.

Nuestros resultados resaltan el importante papel de la etnicidad en la conservación del bosque. La relación entre la variable población indígena y tasa de deforestación fue significativa con signo negativo. Esto fue cierto incluso para aquellos ejidos ubicados cerca de los mercados regionales donde la probabilidad de obtener tasas mayores de deforestación fue significativamente mayor. Es importante mencionar que, si la cohesión social está dada por la composición étnica del grupo, una mayoría étnica no se traduce necesariamente en sustentabilidad. Nuestros resultados indican que en ejidos con población mayormente mestiza la Tasa de deforestación no disminuye como sucedió en ejidos mayormente indígenas.

Los resultados muestran una fuerte relación negativa entre Escolaridad promedio y la variable dependiente, lo cual no es de sorprenderse ya que esta variable se relaciona con la capacidad a nivel comunitario. La capacidad involucra recursos intelectuales, educativos, organizacionales, y culturales que son indispensables para el empoderamiento y desarrollo de la capacidad de autogestión comunitaria (Brown et al., 2007). En proyectos de manejo forestal, la capacidad a nivel comunidad se traduce en mayor poder para negociar términos justos con empresas forestales y exigir rendición de cuentas a las autoridades. La desigualdad de capacidades puede generar una distribución inequitativa del poder y aumentar la marginación de los grupos vulnerables, que pueden ser mujeres o grupos indígenas (Brown et al., 2007; Pérez-Cirera \& Lovett, 2006). Se estima que hasta un $80 \%$ de los bosques comunales en México se encuentran mal manejados debido a la falta de entrenamiento y capacidad técnica (Bray et al., 2005; Larson et al., 2010).

Jefatura de hogar femenina fue significativa, y tuvo un coeficiente $B$ negativo. Bray et al. (2005) consideran que en el medio rural mexicano persisten las prácticas patriarcales y que una población mayoritariamente femenina indica emigración de los hombres. Asimismo, plantean que este fenómeno afecta el tejido social, crea divisiones, reduce el control de la comunidad sobre sus recursos naturales, y aumenta la deforestación. Estas conclusiones no coinciden con nuestra evidencia. Hay experiencias en la región de estudio que sugieren que cuando la jefatura del hogar es ocupada en su mayoría 
por mujeres, éstas adquieren mayor influencia sobre los procesos de toma de decisiones en la comunidad (J.L. Montes, comunicación personal, 21 de abril de 2020). En muchas regiones del mundo, las mujeres son tradicionalmente el principal soporte de las familias rurales y las encargadas de proveer alimentos, agua, y productos forestales no maderables (Brown et al., 2007). De tal forma, es razonable asumir que los roles tradicionales de autoridad, las relaciones de poder, y las prioridades de conservación se modifiquen si aumenta su participación en los procesos de toma de decisiones. En estas circunstancias, podría esperarse que disminuya el interés por las actividades extractivas de madera, que son genéricamente excluyentes al emplear mayoritariamente mano de obra masculina. También es de esperar que se prioricen usos de menor impacto relacionados con la conservación del bosque como el aprovechamiento de productos no maderables, la colecta de plantas medicinales, y la protección de áreas de recarga para manantiales, por ejemplo.

Los análisis no revelaron ninguna relación estadística concluyente entre la variable dependiente y aquellas desarrolladas para representar el capital social estructural (instituciones comunitarias). Nuestra predicción sostenía que la participación en los procesos de toma de decisiones de la asamblea reflejaría el nivel de capital social de las comunidades por medio de las variables de capital social estructural. $\mathrm{Y}$ que éstas, a su vez, serían indicadoras de su capacidad de autogestión (Ostrom, 1990). En los hechos, la asamblea general no es la única estructura sociopolítica que regula la vida social de los ejidos. En muchas comunidades indígenas la gente continúa teniendo más confianza en las autoridades tradicionales para resolver conflictos y tomar decisiones (Heras, 2007). La falta de soporte en los modelos sugiere que la asamblea general tuvo un papel más relevante en ejidos mayormente mestizos.

Las variables con bajo soporte incluyeron Reuniones de asamblea anuales, Reglas formales, Modelo comunitario de extracción y Explotación previa del bosque. Éstas tuvieron coeficientes $B$ negativos, y coincidieron con nuestras predicciones. Otras variables con bajo soporte y coeficientes $B$ positivos fueron Reglas informales, Ingresos por venta de madera e Inventario de madera en pie, pero solo esta última coincidió con nuestras predicciones. La falta de soporte nos impide hacer inferencias acerca de efecto de estas variables a nivel de población, pero las estimaciones y el signo nos ayudan a entender mejor la naturaleza de la relación entre la variable dependiente y los predictores. Más de la mitad de estas variables tienen pendientes $|B| \geq 0.2$, lo que indicaría un efecto moderado sobre la variable dependiente. Un efecto moderado o alto no significativo podría ser el resultado de alta variabilidad en los datos; mientras que baja variabilidad o un tamaño grande de muestra produciría efectos triviales pero significativos.

\section{Limitaciones, recomendaciones y futuras líneas de investigación}

Con relación a los objetivos iniciales, tuvimos éxito comparando los contextos y resultados asociados con el capital social cognitivo, pero no con las instituciones ejidales y el capital social estructural. Consideramos que los resultados pueden servir para hacer extrapolaciones razonables en otras regiones forestales de México, y para entender los procesos de deforestación en otras partes del mundo. Sin embargo, 
dada la diversidad de contextos socio-ecológicos y de propiedad colectiva en otros países, recomendamos cautela para no sobregeneralizar los resultados y hacer predicciones que pudieran ser poco confiables.

En lo que respecta a las recomendaciones, consideramos que la metodología aporta un marco lógico y lecciones para entender los sistemas de gobernanza comunitaria que pueden ser transferibles a otras áreas. Las conclusiones, por su parte, pueden servir para informar programas de desarrollo rural sustentable en México, y como referencia en el diseño de políticas públicas que busquen transferir derechos sobre el territorio y recursos naturales a comunidades en otros países. Por ejemplo, nuestros análisis encontraron soporte para un número de variables o atributos esenciales asociados con el capital social y la gobernanza forestal comunitaria, generando nuevos conocimientos y herramientas para la toma de decisiones. Algunos de estos atributos asociados con el capital social, como género, demografía, y raza, son inamovibles y deben tomarse en cuenta a priori. Otros, como educación, infraestructura, y desarrollo de capacidades pueden atenderse por medio de programas sociales. Un grave error de la Reforma Agraria en México fue superponer los linderos de nuevos ejidos dentro de territorios ancestrales. Esto impuso una estructura institucional distinta a las formas tradicionales de organización de los pueblos indígenas, resultando en una asimilación forzada que afectó a pueblos enteros. En la Sierra Tarahumara, algunos ejidos parte de este estudio fueron creados de esta forma (Wexler \& Bray, 1996). Después de varias décadas, la problemática socio-ecológica persiste y ha dejado como saldo ejidos socialmente fragmentados con altas tasas de deforestación. Los principales afectados han sido las comunidades indígenas, quienes viven en estado de vulnerabilidad permanente al haber sido despojadas de sus derechos constitucionales y de sus bienes materiales e inmateriales (Red en Defensa del Territorio Indígena en la Sierra Tarahumara [REDTDT], 2016).

Para futuras investigaciones, el siguiente paso sería evaluar formalmente el nivel de precisión de nuestros resultados, y extender el alcance geográfico de nuevos análisis. Un mayor tamaño de muestra $N$ permitiría identificar otros atributos esenciales que afectan la gobernanza comunitaria del bosque dentro y fuera del área de estudio (Nagendra, 2007; Ostrom, 2007). Incrementar $N$ también podría aumentar el soporte en los resultados de los modelos, y permitiría emplear un diseño de modelación jerárquica para el análisis de datos. Las comunidades rurales comparten atributos demográficos, sociales y geoespaciales que podrían tratarse como datos anidados. Un diseño jerárquico permitiría reducir el sesgo en las estimaciones dado por la variación asociada a los diferentes niveles en la jerarquía en los datos. Finalmente, pero no por ello menos importante, es importante resaltar el gran vacío de información existente acerca del rol de las mujeres en el manejo del bosque. Futuros trabajos de investigación deben incorporar perspectivas de género para elucidar el papel de las mujeres en las estructuras de gobernanza y los procesos de toma de decisiones en los ejidos y comunidades.

\section{Conclusiones}

Aunque en el área de estudio existen comunidades que han logrado reducir o incluso revertir la deforestación, la pérdida de bosque nativo en décadas 
recientes ha sido significativa. También es notorio que a pesar de la riqueza biológica y cultural de la Sierra Tarahumara, esta región siga siendo una de las menos estudiadas en México. A la fecha solo tenemos conocimiento de dos estudios basados en la valoración sistemática de evidencia empírica y muestras grandes de $N$. Por otra parte, la literatura del manejo forestal comunitario en México está dominada por análisis cualitativos y estudios de caso que no aportan inferencia rigurosa, por lo que su utilidad para hacer predicciones y reproducir casos exitosos a gran escala es limitada. En este sentido, consideramos que nuestro enfoque basado en la inferencia multimodelo, un procedimiento estadístico empleado en numerosas disciplinas científicas (Nichols, 2019), representa un paso en la dirección correcta. Nuestro enfoque analítico nos permitió superar los principales retos que enfrenta el estudio de la gobernanza comunitaria, que son: reducir la incertidumbre asociada con la heterogeneidad de contextos geoespaciales y socio-ecológicos de los datos, y sintetizar información multidisciplinaria y multiescalar (Nagendra, 2007; Ostrom, 2007). Gracias a ello, pudimos confrontar nuestras hipótesis y modelos con los datos, y descubrir nuevos atributos esenciales relacionados con el desempeño de la gobernanza forestal comunitaria que no hubiéramos podido identificar empleando técnicas menos rigurosas. Finalmente, y tomando en cuenta lo importante que puede ser la cohesión social para la estabilidad social, política, y ecológica en zonas rurales, consideramos que las intervenciones y programas públicos deben diseñarse de tal forma que no afecten negativamente las estructuras sociales tradicionales. El desarrollo rural debe potenciar el capital social y humano, fortalecer la capacidad de autogestión, y promover la igualdad de género tomando en cuenta las diferencias étnicas y culturales de cada comunidad. Las comunidades rurales no son homogéneas, y muchas pueden estar profundamente divididas por diferencias étnicas, culturales, y socioeconómicas. Además, pueden contar con niveles bajos de capital social y humano, lo que afectaría su capacidad de organizarse en forma efectiva y su desarrollo. Por ello, la implementación de estrategias de desarrollo rural y manejo forestal comunitario sin una evaluación cuidadosa puede aumentar la deforestación y la pobreza, lo que marginaría aún más a la población indígena y las mujeres (Bray et al., 2005; Brown et al., 2007). No proponemos imponer la conservación comunitaria como única solución a la deforestación y la pérdida de biodiversidad; pero para reducir la pobreza y la degradación ambiental crónica que afecta a las zonas rurales se requieren estrategias que no dependan de programas asistenciales y prácticas burocráticas a corto plazo. Por ello, consideramos que el manejo forestal comunitario, cuyo éxito se basa en el desarrollo efectivo de la capacidad de autogobierno de las comunidades, sea probablemente la mejor alternativa disponible para conservar grandes extensiones de bosques y reducir la pobreza a largo plazo en países como México. 


\section{REFERENCIAS}

Anta, S., \& Pérez, P. (2004). Atlas de experiencias comunitarias en manejo sostenible de los recursos naturales en Oaxaca. Secretaría del Medio Ambiente y Recursos Naturales.

Bray, D. (2013). From Mexico, global lessons for forest governance. Solutions. For a sustainable \& desirable future, 4(3). http://dlc.dlib.indiana.edu/dlc/handle/10535/ 9011

Bray, D., Merino-Pérez, L., \& Barry, D. (2005). The community forests of Mexico. University of Texas Press.

Brown, H. C. P., Lassoie, J. P., \& Wolf, S. A. (2007). An analytic approach to structuring co-management of community forests in Cameroon. Progress in Development Studies, $\quad 7(2), \quad 135-154$. https://doi.org/10.1177\%2F14649934060070 0204

Burnham, K. P., \& Anderson, D. R. (2002). Model selection and multimodel inference: $A$ practical information-theoretic approach (2da ed.). Springer.

Burt, R. S. (2001). Structural holes versus network closure as social capital. En N. Lin, K. Cook, \& R. Burt, Social capital: Theory and research (pp. 31-56). Routledge.

Carrillo-Rubio, E., Kery M., Morreale S. J., Sullivan, P. J., Gardner, B., Cooch, E. G., \& Lassoie, J. P. (2014). Use of multispecies occupancy models to evaluate the response of bird communities to forest degradation associated with logging. Conservation Biology, 28(4), 1034-1044. https://doi.org/ 10.1111/cobi. 12261

Carrillo-Rubio, E. (2005). Conservation in Mexico: From national parks to campesino ecological reserves. En A. Keleman \& I. Angulo (Eds.), Conservation in the matrix: New efforts for inter-disciplinarity in tropical forest conservation (pp. 182-190). Yale School of Forestry \& Environmental Studies.

Coleman, J. S. (1988). Social capital in the creation of human capital. American Journal of Sociology, 94, S95-S121. https://www.jstor.org/stable/2780243

Chhatre, A., \& Agrawal, A. (2009). Trade-offs and synergies between carbon storage and livelihood benefits from forest commons. Proceedings of the National Academy of Sciences of the United States of America (PNAS), 106(42), 17667-17670. https://doi.org/10.1073/pnas.0905308106

Dasgulpta, P., \& Serageldin, I. (1999). Social capital: A multifaceted Perspective. The World Bank.

DiGiano, M., Ellis, E., \& Keys, E. (2013). Changing landscapes for forest commons: Linking land tenure with forest cover change following Mexico's 1992 agrarian counter-reforms. Human Ecology, 41, 707723. https://doi.org/10.1007/s10745-0139581-0 

gobernanza forestal comunitaria: Un análisis cuantitativo. Revista de Sociología, 36(2), 1-20. https://doi.org/10.5354/0719-529X.2021.65567

Dunbar, R. I. M. (1992). Neocortex size as a constraint on group size in primates. Journal of Human Evolution, 22(6), 469-493. https://doi.org/10.1016/0047-2484(92)90081-J

Durán, E., Bray, D. B., Velázquez, A., \& Larrazábal, A. (2010). Multi-scale forest governance, deforestation, and violence in two regions of Guerrero, Mexico. World Development, $\quad 39(4), \quad 611-619$. https://doi.org/10.1016/j.worlddev.2010.08.018

Durán-Medina, E., Mas, J. F., \& Velázquez, V. (2005). Land use/cover change in community-based forest management regions and protected areas in Mexico. In D. Barton, L. Merino-Pérez, \& D. Barry (Eds.), The community forests of Mexico (pp. 215238). University of Texas Press.

Heras, M. (2007). Los raramuri: Una concepción del mundo. PACMYC; Doble Hélice; Instituto Chihuahuense de la Cultura; Gobierno del Estado; CONACULTA.

Hernando, A., Villuendas, D., Vesperinas, C., Abad, M., \& Plastino, A. (2010). Unravelling the size distribution of social groups with information theory in complex networks. The European Physical Journal B, 76, 8797. https://doi.org/10.1140/epjb/e2010-00216-1

Keeley, B. (2007). Human capital: How what you know shapes your life. OECD.

Krishna, A. (2002). Active social capital: Tracing the roots of development and democracy. Columbia University Press.

Krishna A., \& Uphoff, N. (1999). Mapping and measuring social capital: A conceptual and empirical study of collective action for conserving and developing watersheds in Rajasthan, India. (Social capital initiative working paper). The World Bank.

Larson, A. M., Barry, D., Dahal, G. R., \& Colger, C. J. P. (2010). Forest for people: Community rights and forest tenure reform. Routledge.

Lin, N. (2002). Social capital: A theory of social structure and action. Cambridge University Press.

McShane, T. O., Hirsch, P. D., Trung, T. C., Songorwa, A. N., Kinzig, A., Monteferri, B., Mutekanga, D., Van Thang, H., Dammert, J. L., Pulgar-Vidal, M., WelchDevine, M., Brosius, J. P., Coppolillo, P., \& O'Connor, S. (2011). Hard choices: Making trade-offs between biodiversity conservation and human well-being. Biological Conservation 144(3), 966-972. https://doi.org/10.1016/j.biocon.2010.04.038

Nagendra, H. (2007). Drivers of reforestation in human-dominated forests. Proceedings of the National Academy of Sciences of the United States of America (PNAS), 104(39), 15218-15223. https://doi.org/ 10.1073/pnas.0702319104

Nelson, G. C., \& Hellerstein, D. (1997). Do roads cause deforestation? Using satellite images in econometric analysis of land use. American Journal of Agricultural Economics, 79(1), 80-88. https://doi.org/10.2307/1243944

Nichols, J. D. (2019). Confronting uncertainty: Contributions of the wildlife profession to the broader scientific community. The Journal of Wildlife Management, 83(3), 519533. https://doi.org/10.1002/jwmg.21630 
Ostrom, E. (1990). Governing the commons: The evolution of institutions for collective action, Cambridge University Press.

Ostrom, E. (2007). A diagnostic approach for going beyond panaceas. Proceedings of the National Academy of Sciences of the United States of America (PNAS), 104(39), 1518115187. https://doi.org/10.1073/pnas.0702288104

Pérez-Cirera, V., \& Lovett, J. C. (2006). Power distribution, the external environment and common property forest governance: A local user groups model. Ecolical Economics, 59(3), 341-352. https://doi.org/10.1016/j.ecolecon.2005.11.002

Perz, S. G., \& Skole, D. L. (2003). Secondary forest expansion in the Brazilian amazon and the refinement of forest transition theory. Society \& Natural Resources, 16(4), 277-294. https://doi.org/10.1080/08941920390178856

Portes, A. (1998). Social capital: Its origins and applications in modern sociology. Annual Review of Sociology, 24, 1-24. https://doi.org/10.1146/annurev.soc.24.1.1

Poteete, A. R., \& Ostrom, E. (2004). Heterogeneity, group size and collective action: The role of institutions in forest management. Development and Change, 35(3), 435-461. https://doi.org/10.1111/j.14677660.2004.00360.x

Red en Defensa del Territorio Indígena en la Sierra Tarahumara. (2016). Informe: Derechos territoriales de los pueblos indígenas de la Sierra Tarahumara. http://kwira.org/wpcontent/uploads/informeterritoriotarahumara.pdf
Rodrigues, A. S. L., Ewers, R. M., Parry, L., Souza, C., Veríssimo, A., \& Balmford, A. (2009). Boom-and-bust development patterns across the Amazon deforestation frontier. Science, 324(5933), 1435-1437. https://doi.org/10.1126/science.1174002

Rzedowski, J. (2006). Vegetación de México (1ra. ed.). Comisión Nacional para el Conocimiento y Uso de la Biodiversidad.

Sarukhárn, J., \& Larson, J. (2001). When the commons become less tragic: Land tenure, social organization, and fair trade in Mexico. En J. Burger, E. Ostrom, R. Norgaard, D. Policansky, \& B. D. Goldstein (Eds.), Protecting the commons: A framework for resource management in the Americas (pp. 45-50). Island Press.

Sorensen, C. (2000). Social capital and rural development: A discussion of issues. The World Bank.

Toledo, V. M. (2000). La paz en Chiapas: Ecología, luchas indígenas y modernidad alternativa. Ediciones Quinto Sol.

Tornos, J., Arroyo, A. Martínez, M., \& López, J. (2012). Transparencia, rendición de cuentas y participación: Una agenda común para la cohesión social y la gobernanza en América Latina. Diputación de Barcelona.

Weaver, T. (2000). Changes in forestry policy, production, and the environment in Northern Mexico: 1960-2000. Journal of Political Ecology, 7(1), 1-18. https://doi.org/10.2458/v7i1.21544 
Carrillo-Rubio, E., Morreale, S. J., \& Pérez-Cirera, V. (2021). Impacto positivo del capital social en la gobernanza forestal comunitaria: Un análisis cuantitativo. Revista de Sociología, 36(2), 1-20. https://doi.org/10.5354/0719-529X.2021.65567

Westholm, L., Biddulph, R., Hellmark, I., \& Exbom, A. (2011). REDD+ and tenure: $A$ review of the latest developments in research, implementation and debate. https://bit.ly/3mgUj29

Wexler, M., \& Bray, D.B. (1996). Reforming forests: From community forests to corporate forestry in Mexico. In L. Randall (Ed.), Reforming Mexico's agrarian reform (pp. 235-245). University of Texas Press.
Zuur, A. F., Ieno, E. N., Walker, N., Saveliev, A. A., \& Smith, G. M. (2009). Mixed effects models and extensions in ecology with $R$. Springer.

Manuscrito recibido: 19-07-2021

Manuscrito aceptado: 04-10-2021 